\title{
High Expression of Matrix Metalloproteinase-11 indicates Poor Prognosis in Human Cholangiocarcinoma
}

\author{
Taweesak Tongtawee ${ }^{1}$, Soraya J Kaewpitoon ${ }^{2,3}$, Ryan Loyd ${ }^{2,3}$, Supachai \\ Chanvitan $^{4}$, Kawin Leelawat ${ }^{4,5}$, Niphol Praditpol ${ }^{6}$, Supathip Jujinda ${ }^{4}$, Natthawut \\ Kaewpitoon $^{3,7 *}$
}

\begin{abstract}
Background: Cholangiocarcinoma (CHCA) is serious public health problem in Thailand, especially in the northeastern and northern regions. CHCA is known as one of the most aggressive malignant tumors associated with local invasion and a high rate of metastasis. A crucial step in the invasion process is the proteolytic degradation of the extracellular matrix (ECM) and basal membranes, for which several studies have shown a critical role played by matrix metalloproteinase-11 (MMP-11). Objective: This study aim to detect MMP-11 expression in CHCA specimens and any correlation with survival time. Materials and Methods: A retrospective analysis was conducted of 30 patients with CHCA in Rajvithi hospital, who had undergone immunohistochemical staining of MMP-11. Relationships between clinicopathological data and MMP-11 expression in CHCA specimens were analyzed by the $\chi 2$ test or Fisher's exact test. The estimated survival and the survival differences were analyzed by the Kaplan-Meier method and the log-rank test, respectively. Results: MMP-11 expression was found in 15 specimens (50\%). The overall mean survival time is 237.0 days (95\% CI 135.4-338.5, SD 271 .9). Specimens with a positive MMP-11 had an average survival time of 136.7 days ( $95 \%$ CI 50.3-223.1, SD 156.0). Survival differences was signficant for the positive and negative MMP-11 $(\mathrm{p}=0.022)$, but not well differentiated tumor and moderate to poor differentiated tumor ( $\mathrm{p}=\mathbf{0 . 7 5 5}$ ), CA19-9 level of $>1,000$ and $<1,000(\mathrm{p}=0.488)$, and between advanced and non-advanced staging $(\mathrm{p}=\mathbf{0 . 3 8 8})$. Conclusions: The positive MMP-11 expression indicates poor prognosis in CHCA specimens.
\end{abstract}

Keywords: Cholangiocarcinoma - matrix metalloproteinase-11 - expression - prognosis

Asian Pac J Cancer Prev, 16 (9), 3697-3701

\section{Introduction}

Cholangiocarcinoma (CHCA); the bile duct cancer, is known as one of the most aggressive malignant tumors associated with local invasiveness and a high rate of metastasis. It is also known to be one of the most common causes of cancer related death in Thailand (Green et al., 1991; Shin, et al., 2010). The experimental and epidemiological evidences strongly indicated that the liver fluke infection in the etiology of CHCA (Thamavit, et al., 1978; IARC, 1994; Sripa, et al., 2007). CHCA is reported frequently in Thailand, and found the highest of the world (Sripa, et al., 2007; Kaewpitoon, et al., 2008a). Three-year survival rates of $35 \%$ to $50 \%$ are achieved only in a subset of patients who have negative histological margins at the time of surgery (Akamatsu N, et al., 2011). Survival of CHCA patients in northeastern Thailand after supportive treatment was reported and indicated that the stage of disease was an important prognosis factor affecting survival of CHCA patients who had diagnosis in late stage. The encourage patients to see health personnel at early stage is very important (Thunyaharn et al., 2013). Palliative therapeutic approaches, consisting of percutaneous and endoscopic biliary drainage, have usually been used for these patients because there is no effective chemotherapeutic treatment for this type of cancer. Therefore, identification of the molecules involved in CHCA cell invasion is crucial for the development of novel drug treatments for this disease. Tumor invasion is a complex process that requires interaction between the invasive cells and the extracellular matrix (ECM). A crucial step in invasive processes is the proteolytic degradation of the ECM and basal membranes. Among the enzymes responsible for ECM degradation, several studies have shown a critical role played by matrix metalloproteinase (MMPs) (Curran and Murray, 1999; Amalinei, et al., 2010). MMP are zinc-dependent endopeptidases, which are collectively capable of degrading all constituents

${ }^{1}$ Department of surgery, ${ }^{2}$ Department of Family and Community Medicine, ${ }^{3}$ Parasitic Disease Research Unit, Institute of Medicine, Suranaree University of Technology, ${ }^{7}$ Faculty of Public Health, Vongchavalitkul University, Nakhonratchasima, ${ }^{4}$ Department of Surgery, ${ }^{5}$ Department of Pathology, ${ }^{6}$ Immunohistochemistry Section, Rajvithi Hospital, Bangkok, Thailand * For correspondence: natthawut.k@hotmail.com,natthawut_kae@vu.ac.th 
of the ECM. The 21 distinct MMPs so far identified, can be grouped, on the basis of their structure and substrate specificity, into the following subclasses: (a) collagenases (MMP-1, 8, 13), (b) gelatinases (MMP-2, 9), (c) stromelysins (MMP-7 or matrilysin, 3 or ST-1, 10 or ST-2, 11 or stromelysin-3), (d) membrane-type MMPs (MT1-, MT2-, MT3-, MT4-, MT5-MMP) and other MMPs. Disposing overlapping yet distinct functions, as a whole, they can degrade practically every extracellular matrix component, acting always under a multistep regulatory system including specific tissue inhibitors of metalloproteinase and inducers (e.g., extracellular matrix metalloproteinase inducer) (Curran S and Murray, 1999; Amalinei et al., 2010; Bourboulia and Stetler-Stevenson, 2010; Gialeli et al., 2011). Previous study demonstrated that CHCA cells that were cultured with Matrigel (the component of extracellular matrix basement membrane) expressed many genes involved in cancer progression (Keeratichamroen et al., 2011). MMP-11 is found to upregulated more than three folds in this cell line. MMP-11 in particular, has been originally described and isolated from fibroblastic cells surrounding invasive breast carcinoma (Basset et al., 1993; Basset et al., 1994; Rouyer et al., 1994). Ever since, it remains an intriguing target for further study due to the fact that it is persistently found over expression in almost every aggressive malignancy, although its putative mature forms seem to exhibit little or no obvious proteolytic activity towards major extracellular matrix components. Its predicted structure shares common features with that of other stromelysins and collagenases. However, unlike all other MMPs, which are secreted in proenzyme form, stromelysin-3 along with membrane type 1-MMP, are activated before secretion by Golgiassociated furin-like proteases (Peruzzi et al., 2009; Brasse et al., 2010). The role of MMP-11 in cancer progression has been shown by several preclinical observations: its expression promotes tumor take in mice (Brasse D, et al., 2010), homing of malignant epithelial cells (Delany AM and Canalis E, 2001), cancer progression by remodeling extracellular matrix, and anti-apoptotic and anti-necrotic effect on tumor cells (Fromigue et al., 2003). MMP-11 deficiency increases tumor-free survival and modulates local or distant invasion (Noel et al., 1996); knockdown of MMP-11 mRNA in gastric cancer cells suppresses tumor growth in vitro and in vivo (Deng et al., 2005). In addition, expression of MMP-11 is significantly associated with survival in prostatic adenocarcinoma. High levels may potentially be used for prediction of a poor prognosis (Nonsrijun et al., 2013). However, until now there is no publication about the roles of MMP-11 expression in CHCA specimens. In this study, detection of MMP-11 expression in CHCA specimens and analyzed the relationship between expression MMP-11 and survival time.

\section{Materials and Methods}

A retrospective analysis was performed, of 30 specimens of patients with CHCA in Rajvithi hospital who had undergone immunohistochemical staining of MMP-11. Eligible participants were CHCA patients who received treatment in Rajvithi hospital between January 2009 and September 2012. CHCA specimens were fixed in paraffin blocks and all of the specimens had the diagnosis confirmed by a pathologist. We analyzed the patient's demographic data, the types of CHCA, tumor staging, histological differentiation and level of CA 19-9 from the medical records. The study was approved by the medical ethics committee of Rajavithi hospital (Ethics no. 161/2554).

\section{Immunohistochemistry}

The slides were baked at $60^{\circ} \mathrm{C}$ for two hours, deparaffinized with xylene, and then rehydrated. The sections were submerged into $10 \mathrm{mmol}$ citric buffer $(\mathrm{pH}$ 6.0) and microwaved for antigen retrieval, after which they were treated with $3 \%$ hydrogen peroxide in methanol to quench endogenous peroxidase activity. Sections were then incubated in $1 \%$ bovine serum albumin to block nonspecific bindings. Sections were incubated with rabbit monoclonal anti-MMP-11 (1:100; ABCAM USA) over night at $4^{\circ} \mathrm{C}$. Normal goat serum was used as a negative control. After washing, the tissue sections were treated with horseradish peroxidase antibody. Tissue sections were then counter-stained with hematoxylin, dehydrated, and mounted.

MMP-11 positive cells stained light brown. The degree of immunostaining was independently reviewed and scored by two observers based on the proportion of positively stained tumor cells and on the intensity of staining (18).Tumor cells proportion was scored as follows: 0 ( $\leq 5 \%$ positive tumor cells), 1 (6\%-25\% positive tumor cells), 2 (26\%-50\% positive tumor cells) , and 3 ( $>50 \%$ positive tumor cells). Staining intensity was graded according to the following criteria: 0 (no staining), 1 (weak staining=light yellow), 2 (moderate staining=yellow brown), and 3 (strong staining=brown). The staining index was calculated as the product of staining intensity score and the proportion of positive tumor cells. Using this method of assessment, we evaluated the levels of MMP11 in cholangiocarcinoma specimens by determining the staining index with scores of $0,1,2,3,4,6$, or 9 . The cut off value for high and low expression levels were chosen based on a measure of heterogeneity using the log-rank test with respect to the overall survival. An optimal cut off value was identified: a staining index score of 4 or higher was used to define tumors with high levels of MMP-11 expression, and a staining index score of 3 or lower was used to indicate low levels of MMP-11 expression.

\section{Statistical analysis}

The normality of the distribution of quantitative variable was assessed by the Kolmogorov-Smirnov test. Results are expressed as arithmetic mean \pm standard deviation except when specifically noted. Statistical analysis of the association between clinicopathological data and the expression of Matrix Metalloproteinase (MMP-11) in cholangiocarcinoma specimens were performed using the Fisher's exact test. The Kaplan-Meier method was used to estimate survival as a function of time, and the survival differences were analyzed by logrank test. A p-value of $<0.05$ was considered statistically 
significant. SPSS version 16.0.0 (SPSS Inc, Chicago,IL) was used for the statistical analysis.

\section{Results}

A total of 30 patients with CHCA were selected from January 2009 through to September 2012. The age range was between 44 and 78 years of age. There were $15(50 \%)$ males and 15 (50\%) females. MMP-11 expression in CHCA were characterized by immunochemistry staining, were showed in Figure 1. A baseline characteristic of positive and negative groups shown in Table 1, and survival time is shown in Figure 2.

In the survival analysis, the overall mean survival time is 236.97 days (95\% CI 135.44-338.49, SD 271.88). Specimens with a positive MMP-11 had an average survival time of 136.73 days (95\%CI 50.33-223.13,SD

Table 1. Baseline Characteristics of Positive and Negative Groups

\begin{tabular}{|c|c|c|c|}
\hline \multirow[t]{2}{*}{ Features } & \multirow[t]{2}{*}{$\begin{array}{l}\text { Total } \\
\mathrm{n}(\%)\end{array}$} & \multicolumn{2}{|c|}{$\begin{array}{c}\text { MMP-11expression } \\
\mathrm{n}(\%)\end{array}$} \\
\hline & & Positive & Negative \\
\hline \multicolumn{4}{|l|}{ Gender } \\
\hline Male & $15(50)$ & $8(26.7)$ & $7(23.3)$ \\
\hline Female & $15(50)$ & $7(23.3)$ & $8(26.7)$ \\
\hline \multicolumn{4}{|c|}{ Histological differentiation } \\
\hline $\begin{array}{l}\text { Well differentiation } \\
\text { differentiation }\end{array}$ & $21(70)$ & $9(30)$ & $12(40)$ \\
\hline Moderate to poor & $9(30)$ & $6(20)$ & $3(10)$ \\
\hline \multicolumn{4}{|l|}{ CA 19-9 } \\
\hline$>1000$ & $11(36.7)$ & $6(20)$ & $5(16.7)$ \\
\hline$<1000$ & $19(63.3)$ & $9(30)$ & $10(33.3)$ \\
\hline \multicolumn{4}{|l|}{ Type of cancer } \\
\hline Intrahepatic & $24(80)$ & $13(43.3)$ & 11(36.6) \\
\hline Extrahepatic & $6(20)$ & $2(6.67)$ & $4(13.3)$ \\
\hline \multicolumn{4}{|l|}{ Staging } \\
\hline Non-advanced & $13(43.3)$ & $2(6.67)$ & 11(37.6) \\
\hline Advanced & $17(56.6)$ & $13(43.3)$ & $4(13.3)$ \\
\hline \multicolumn{4}{|l|}{ Status of patients } \\
\hline Dead & $7(23.3)$ & $5(16.7)$ & $2(6.6)$ \\
\hline Alive & $23(76.7)$ & $2(6.6)$ & $13(43.3)$ \\
\hline
\end{tabular}
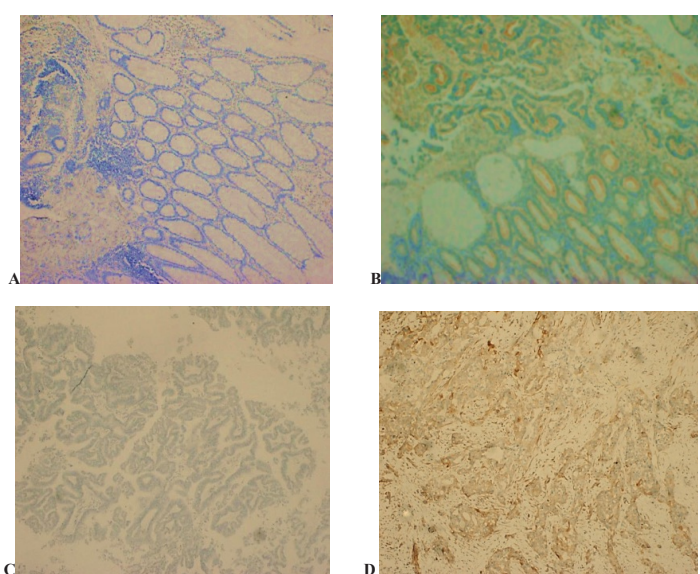

Figure 1. Expression of Matrix Metalloproteinase11(MMP-11) in CHCA by immunochemistry staining. A; Normal mucosa of gastric cancer (Control), B; Expression of MMP-11 in gastric cancer tissue (Control), C; Negative MMP11 expression in CHCA tissue, D; Positive MMP-11 expression in CHCA tissue
156.019). Patients with positive MMP-11and negative MMP-11 had different survival times $(\mathrm{p}=0.022$; Figure 3). Patients with tumor staging, level CA19-9 and

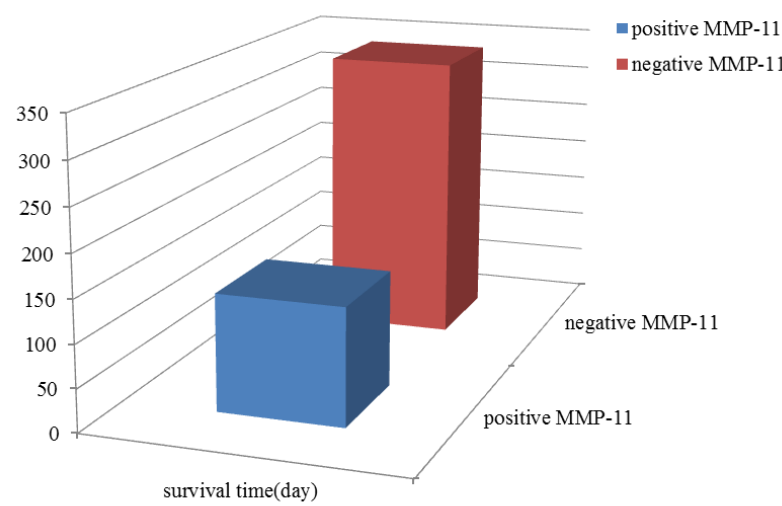

Figure 2. Survival Time in CHCA Patients with Positive and Negative MMP-11

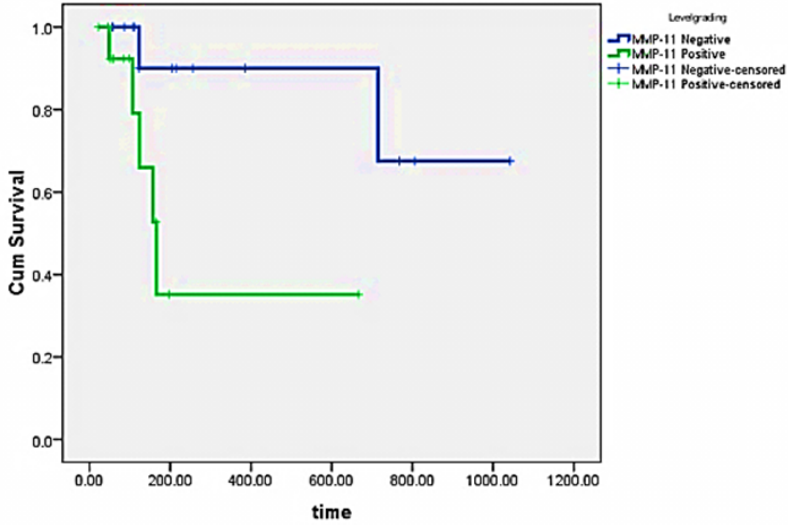

Figure 3. Survival Analysis of Expression MMP-11 and Survival Time

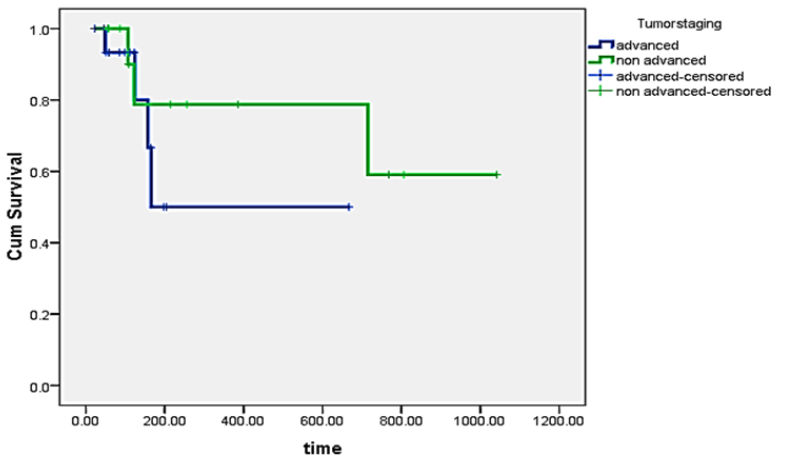

Figure 4. Survival Analysis of Staging of CHCA and Survival Time

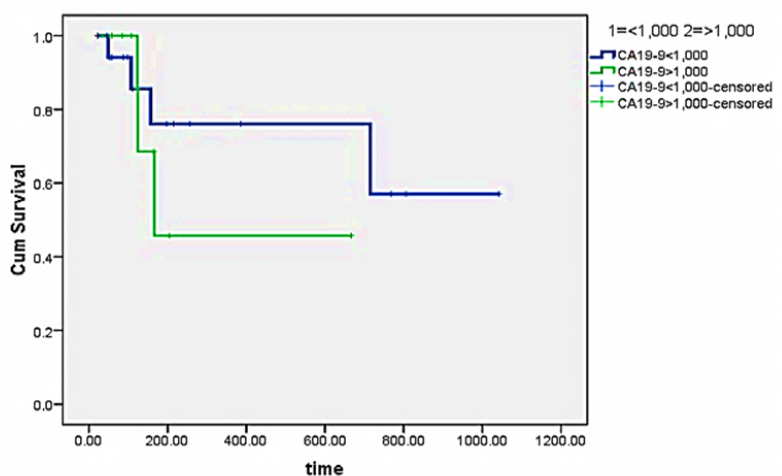

Figure 5. Survival Analysis of Level CA19-9 and Survival Time

Asian Pacific Journal of Cancer Prevention, Vol 16, 2015 


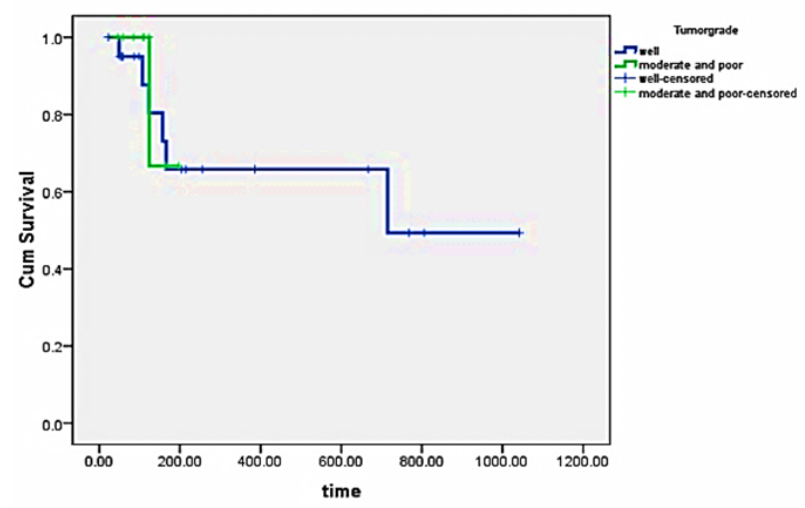

Figure 6. Survival Analysis of Histological Differentiated and Survival Time

histopathological differentiated had no different in the survival time ( $\mathrm{p}=0.338$; Figure $4, \mathrm{p}=0.488$; Figure 5 and $\mathrm{p}=0.755$; Figure 6 , respectively).

\section{Discussion}

CHCA is still a major public health problem in Thailand, especially in the northeastern and northern region (Sripa et al., 2007; Kaewpitoon et al., 2008a). Identification of the molecules involved in CHCA cell invasion is crucial for the development of novel drug treatments for this disease. Tumor invasion is a complex process that requires interaction between the invasive cells and the extracellular matrix (ECM). A crucial step in invasive processes is the proteolytic degradation of the ECM and basal membranes. Among the enzymes responsible for ECM degradation, several studies have shown a critical role played by MMPs (Curran and Murray, 1999; Amalinei et al., 2010). The MMPs are a large family of proteolytic enzymes, which are involved in the degradation of many different components of the extracellular matrix. There is increasing evidence to indicate that individual MMPs have important roles in tumor invasion and metastasis (Bourboulia and Stetler-Stevenson, 2010; Gialeli et al., 2011). MMP-11 or stromelysin-3 was first characterized from stromal cells of breast cancer (Basset et al., 1990). It was unique among the MMPs since it was shown in stromal fibroblast cells adjacent to tumor cells, rather than within tumor cells themselves (Basset et al., 1990; 1993). Previous studies demonstrated that CHCA cells that were cultured with Matrigel (the component of extracellular matrix of basement membrane) expressed many genes involved in cancer progression (Keeratichamroen et al., 2011). MMP-11 is found to up-regulated more than three folds in this cell line. Nonsrijun et al. (2013) has been reported that MMP-11 is significantly associated with survival in prostatic adenocarcinoma. Subsequently, they found that patients with low-level MMP-11 expression had significantly longer survival time compared with high levels of MMP-11. Recent study is the first study of the role of MMP-11 expression in CHCA specimens. In this study, it is shown that CHCA patients who had positive MMP-11 has a statistic significant of survival time lower than negative MMP-11. In general, prognosis of CHCA still involves many factors, for example staging of the tumor, histological differentiation and the level of CA19-9 which effects the survival time. However, we cannot evaluate the relationship between expression MMP-11 and prognostic factors of CHCA because of a small sample size in this study. One limitation to this study is that although there are a large number of patients with CHCA at Rajvithi Hospital, but most patients did not undergo surgical treatment because of the advanced stage to presentation. Therefore, we were not able to perform histopathological study from it.

In conclusion, this study indicates that positive MMP11 expression in CHCA specimens represent a poorer prognosis than negative MMP-11 expression.

\section{Acknowledgements}

We would like to thanks Suranaree University of Technology for support a grant. Special thanks to Prof.Dr.Prasit Pengsaa for his valuable comment and suggestion.

\section{References}

Akamatsu N, Sugawara Y, Hashimoto D (2011). Surgical strategy for bile duct cancer: advances and current limitations. World $J$ Clin Oncol, 10, 94-107.

Amalinei C, Caruntu ID, Giusca SE, et al (2010). Matrix metalloproteinases involvement in pathologic conditions. Rom J Morphol Embryol, 51, 215-28.

Basset P, Bellocq JP, Wolf C, et al (1990). A novel Metalloproteinase gene specifically expressed in stromal cells of breast carcinomas. Nature, 348, 699-704.

Basset P, Wolf C, Chambon P (1993). Expression of the stromelysin-3 gene in fibroblastic cells of invasive carcinomas of the breast and other human tissues: a review. Breast Cancer Res Treat, 24, 185-93.

Basset P, Wolf C, Rouyer N, et al (1994). Stromelysin-3 in stromal tissue as a control factor in breast cancer behavior. Cancer, 74, 1045-9.

Bourboulia D, Stetler-Stevenson WG (2010). Matrix metalloproteinases (MMPs) and tissue inhibitors of metalloproteinases (TIMPs): Positive and negative regulators in tumor cell adhesion. Semin Cancer Biol, 20, 161-8.

Brasse D, Mathelin C, Leroux K, et al (2010). Matrix metalloproteinase 11/stromelysin-3 exerts both activator and repressor functions during the hematogenous metastatic process in mice. Int J Cancer, 127, 1347-55.

Curran S, Murray GI (1999). Matrix metalloproteinases in tumour invasion and metastasis. J Pathol, 189, 300-8.

Delany AM, Canalis E (2001). The metastasis-associated metalloproteinase stromelysin-3 is induced by transforming growth factor-beta in osteoblasts and fibroblasts. Endocrinology, 142, 1561-6.

Deng H, Guo RF, Li WM, et al (2005). Matrix metalloproteinase 11 depletion inhibits cell proliferation in gastric cancer cells. Biochem Biophys Res Commun, 326, 274-81.

Fromigue O, Louis K, Wu E, et al (2003). Active stromelysin-3 (MMP-11) increases MCF-7 survival in three-dimensional Matrigel culture via activation of p42/p44 MAP-kinase. Int J Cancer, 106, 355-63.

Gialeli C, Theocharis AD, Karamanos NK (2011). Roles of matrix metalloproteinases in cancer progression and their pharmacological targeting. FEBS J, 278, 16-27.

Green A, Uttaravichien T, Bhudhisawasdi V, et al (1991). Cholangiocarcinoma in north east Thailand. A hospital-based study. Trop Geogr Med, 43, 193-8. 
IARC (1994). Infection with liver flukes (Opisthorchis viverrini, Opisthorchis felineus and Clonrochis sinensis). IARC Monogr Eval Carcinog Risks of Hum, 61, 121-75.

Kaewpitoon N, Kaewpitoon SJ, Pengsaa P, et al (2008). Opisthorchis viverrini: the carcinogenic human liver fluke. World J Gastroenterol, 7, 666-74.

Keeratichamroen S, Leelawat K, Thongtawee T, et al (2011). Expression of CD24 in cholangiocarcinoma cells is associated with disease progression and reduced patient survival. Int J Oncol, 39, 873-81.

Linder C, Engel G, Auer G, et al (1997). Distribution of stromelysin-3 mRNA transcripts and microvessels in human breast carcinomas. Breast Cancer Res Treat, 42, 207-13.

Noel AC, Lefebvre O, Maquoi E, Vet al (1996). Stromelysin-3 expression promotes tumor take in nude mice. J Clin Invest, 97, 1924-30.

Nonsrijun N, Mitchai J, Brown K, et al (2013). Expression of MMP-11 is significantly associated with survival in prostatic adenocarcinoma. High levels may potentially be used for prediction of a poor prognosis. Asian Pac J Cancer Prev, 14, 3331-5.

Peruzzi D, Mori F, Conforti A, et al (2009). MMP11: a novel target antigen for cancer immunotherapy. Clin Cancer Res, 15, 4104-13.

Rouyer N, Wolf C, Chenard MP, et al (1994). Stromelysin-3 gene expression in human cancer: an overview. Invasion Metastasis, 14, 269-75.

Shin HR, Oh JK, Masuyer E, et al (2010). Epidemiology of cholangiocarcinoma: an update focusing on risk factors. Cancer Sci, 101, 579-85.

Sripa B, Kaewkes S, Sithithaworn P, Met al (2007). Liver fluke induces cholangiocarcinoma. PLoS Med, 4, e201.

Thamavit W, Bhamarapravati N, Sahaphong S, etal (1978). Effects of dimethylnitrosamine on induction of cholangiocarcinoma in Opisthorchis viverrini-infected Syrian golden hamsters. Cancer Res, 38, 4634-9.

Thunyaharn N, Promthet S, Wiangnon S, Set al (2013). Survival of cholangiocarcinoma patients in northeastern Thailand after supportive treatment. Asian Pac J Cancer Prev, 14, 7029-32.

Zhao ZS, Chu YQ, Ye ZY, et al (2010). Overexpression of matrix metalloproteinase 11 in human gastric carcinoma and its clinicopathologic significance. Hum Pathol, 41, 686-96. 